\title{
Passage repetition and presentation time in connected discourse learning
}

\author{
DAVID J. KING \\ Oregon State University, Corvallis, Oregon 97331
}

\begin{abstract}
Subjects learned a 25-word passage of connected discourse. The learning conditions consisted of a factorial combination of three total presentation times $(30,45$, and $60 \mathrm{sec})$ and three conditions of repetition (one, three, and five). Significant presentation time effects were produced because of the extremely rapid rate of presentation under the five-repetition condition. This condition also masked the previously found superiority of the repetition effect.
\end{abstract}

Two previous studies (King, 1975, 1978) demonstrated the superiority of a repetition vs. no repetition condition in the learning of a short, two-sentence passage of connected discourse. The advantage of the repetition effect was demonstrated when the presentation time was either confounded or varied factorially with repetitions. This certainly does not support at least one version of the total-time hypothesis, which states "a fixed amount of time is necessary to learn a fixed amount of material regardless of the number of individual trials into which that time is divided" (Cooper \& Pantle, 1967, p. 221). Neither of the above-cited studies approached such a rapid presentation rate that might result in an increase in the significance of the time dimension. The present study addresses itself to this question.

\section{METHOD}

This study is a systematic replication of King (1978). The learning material was the same 25-word passage of two sentences. The passage read: "The early morning sun shining through the yellow curtain gave the bedroom a translucent quality. George opened one eye and quickly closed it in disgust." The learning material was prepared on 16-mm film as follows. Each frame of the film contained one word with no blank frames between words. After completion of the passage, a blank frame was inserted and the process continued until the material was repeated five times.

The general design of the study was a 2 by 3 by 3 factorial. Sex was the dichotomous dimension, the number of repetitions (one, three, and five) was the second dimension, and the total presentation time $(30,45$, and $60 \mathrm{sec})$ was the third dimension. Each cell contained 5 individuals, for a total of 90 subjects. A total of 95 subjects were used, as data from 5 subjects were discarded for failure to learn the material within a reasonable amount of time. The leaming material was presented on a motion-analysis projector that allowed control of the time per frame to accommodate the nine conditions of presentation time and number repetitions. Subjects were assigned at random to the nine treatment conditions, within the limitations of numerical balance. Following the presentation of each treatment condition, the subjects wrote their recalls "as close to the original as possible." Subjects were run one at a time, with trials continuing to a criterion of one perfect recall.

\section{RESULTS AND DISCUSSION}

The number of trials to criterion and the total learning time (the time, in seconds, from the start of the first presentation to the completion of a perfect recall) were the dependent variables. In submitting both dependent variables to a 2 by 3 by 3 ANOVA, there was only one significant effect. There was a significant effect $[F(2,72)=10.88, p<.01]$ of presentation time on the trials-to-criterion measure. The mean number of trials to criterion for the 30-, 45-, and 60-sec presentation times, respectively, were $4.0,2.9$, and 2.3 . These results are very different from the prior study (King, 1978), in which the repetition effect was significant for both variables and presentation time was not significant. On the surface, the data support the total-time hypothesis, with greater amounts of presentation time resulting in more rapid learning. If this were the case, however, it would be logical to expect an appropriately significant finding in the total learning time variable. That is, as presentation time increases, the total learning time should decrease. The means do decrease as expected (i.e., $487.5,436.4$, and 374.1 ), but the variability is so large that the $F$ ratio does not reach significance.

Of greater concern and interest was the failure of the repetition effect on both variables. The two previous studies showed a strong and consistent effect. In the analysis that follows, I address only the total learning time, although the same results were found with the trials-to-criterion measure. While there is not a statistically significant interaction effect between repetitions and presentation time (again, because of variability), the result of the interaction is very obvious when viewed in Figure 1.

The repetition effect is present through three repetitions at all presentation times. With five repetitions, however, the 30- and 45-sec presentation times are so rapid as to result in an actual increase in total learning time. Presentation rates of around 3-4 frames/sec are 


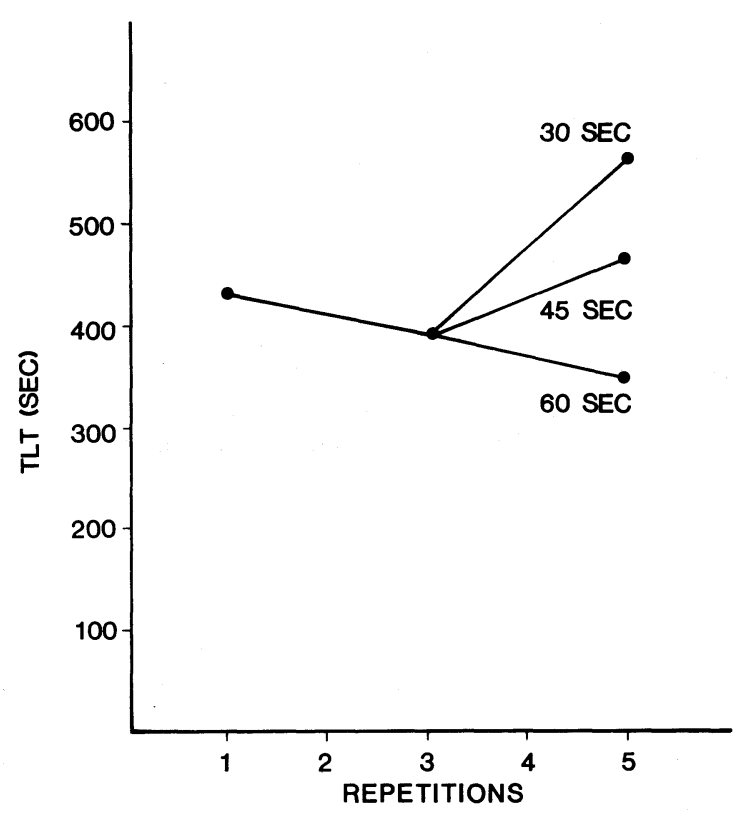

Figure 1. Total learning time as a function of number of repetitions and total presentation time. simply too fast to facilitate learning through repetition and rehearsal. This finding also suggests why there were significant presentation time effects (unlike previous studies). Presentation time is significant in this study because we have approached an extreme rate outside of that broad range of rates where there appears to be little relationship between presentation rate and total learning time (King, 1974, Figure 1).

\section{REFERENCES}

Cooper, E. H., \& Pantle, A. J. The total-time hypothesis in verbal learning. Psychological Bulletin, 1967, 68, 221-234.

KING, D. J. Total presentation time and total learning time in connected discourse learning. Journal of Experimental Psychology, 1974, 103, 586-589.

KING, D. J. The influences of repetition vs. no repetition given equal presentation time on the learning of connected discourse. Bulletin of Psychonomic Society, 1975, 5, 501-503.

KING, D. J. The influence of passage repetition and presentation time on the learning of connected discourse. Bulletin of Psychonomic Society, 1978, 12, 229-230.

(Received for publication June 13, 1979.) 